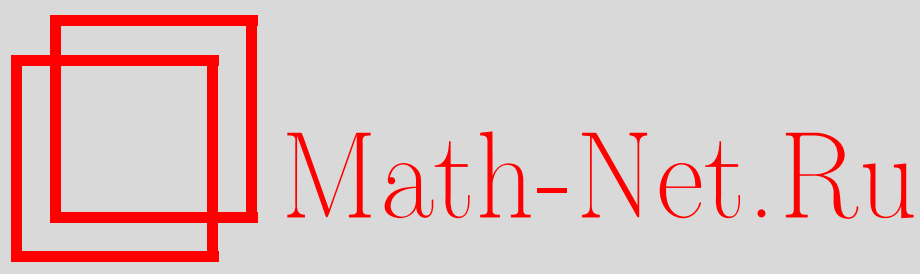

Ф. М. Мухамедов, О равномерных эргодических теоремах для квадратичных процессов на $C^{*}$-алгебрах, Maтем. сб., 2000, том 191, номер 12, 141-152

DOI: https://doi.org/10.4213/sm532

Использование Общероссийского математического портала Math-Net.Ru подразумевает, что вы прочитали и согласны с пользовательским соглашением

http: //www. mathnet.ru/rus/agreement

Параметры загрузки:

IP : 54.162 .27 .143

26 апреля 2023 г., 16:04:12 


\author{
Ф.М. Мухамедов
}

\title{
О равномерных эргодических теоремах для квадратичных процессов на $C^{*}$-алгебрах
}

\begin{abstract}
В работе исследуются вопросы сходимости временных средних по Чезаро стационарных квантовых квадратичных процессов в равномерной топологии, определенных на $C^{*}$-алгебрах.

Дается необходимое и достаточное условие сходимости средних в равномерной топологии. Кроме того, исследуется сходимость средних с весом в равномерной топологии. Доказана сходимость средних с весом $\Phi$-функций Безиковича.

Библиографиия: 15 названий.
\end{abstract}

Квадратичные операторы как объект исследования появились в работах Бернштейна. В [1], например, рассматривалось предельное поведение траекторий таких операторов (эта задача естественньм образом возникает при изучении некоторых моделей со взаимодействиями). Одной из задач теории квадратичных операторов является изучение их эргодических свойств. Этим вопросам посвяшены, например, работы [2]-[5]. В работах [6], [7] исследовались квантовые (некоммутативные) квадратичные процессы, определенные на алгебрах фон Неймана, и изучались некоторые эргодические свойства таких процессов.

В настоящей работе рассматриваются вопросы сходимости временных средних по Чезаро стационарных квантовых квадратичных процессов в равномерной топологии, определенных на $C^{*}$-алгебрах.

В $\S 1$ дается необходимое и достаточное условие сходимости средних в равномерной топологии. В $\S 2$ исследуется сходимость средних с весом в равномерной топологии. Доказана сходимость средних с весом $\Phi$-функций Безиковича.

Автор приносит благодарность рецензенту за полезные замечания.

\section{$\S 1$. Равномерная эргодическая теорема}

Пусть $\mathfrak{A}-C^{*}$-алгебра с единицей. Через $\beta$ обозначим наименьшую $C^{*}$-кросснорму на $\mathfrak{A} \odot \mathfrak{A}$, существование которой доказано в [8; предложение 1.22 .2 , теорема 1.22.6]. Далее, пусть $\mathfrak{A} \otimes \mathfrak{A}$ обозначает пополнение $\mathfrak{A} \odot \mathfrak{A}$ по норме $\beta$, для которой будет использован обычный символ $\|\cdot\| ; S$ и $S^{2}$ обозначают совокупность всех состояний на $\mathfrak{A}$ и $\mathfrak{A} \otimes \mathfrak{A}$ соответственно. Введем оператор $U: \mathfrak{A} \otimes \mathfrak{A} \rightarrow \mathfrak{A} \otimes \mathfrak{A}$, который для элементов вида $x \otimes y$ определяется как $U(x \otimes y)=y \otimes x$ и продолжается на все $\mathfrak{A} \otimes \mathfrak{A}$ по линейности с сохранением нормы.

ОПРЕДЕЛЕниЕ 1.1. Пара $\left(P^{(t)}, \varphi\right)$, где $P^{(t)}: \mathfrak{A} \rightarrow \mathfrak{A} \otimes \mathfrak{A}, t \geqslant 1,-$ семейство линейных отображений, а $\varphi \in S$ - состояние, называется квантовым квадратичнылм стохастическим прочессом (к.к.с.п.), если она удовлетворяет следующим условиям: для каждого $t \geqslant 1$

(i) $P^{(t)} 1_{\mathfrak{A}}=1_{\mathfrak{A} \otimes \mathfrak{A}}$, где $1_{\mathfrak{A}}$ и $1_{\mathfrak{A} \otimes \mathfrak{A}}-$ единицы алгебр $\mathfrak{A}$ и $\mathfrak{A} \otimes \mathfrak{A}$ соответственно; 
(ii) $P^{(t)}\left(\mathfrak{A}_{+}\right) \subset(\mathfrak{A} \otimes \mathfrak{A})_{+}$, где $\mathfrak{A}_{+}$и $(\mathfrak{A} \otimes \mathfrak{A})_{+}-$положительные части $\mathfrak{A}$ и $\mathfrak{A} \otimes \mathfrak{A}$ соответственно;

(iii) $P^{(t)}(\mathfrak{A}) \subset\{x \in \mathfrak{A} \otimes \mathfrak{A}: U(x)=x\}$;

(iv) для $s \geqslant 1$ имеет место

$$
P^{(t+s)} x=\left(P^{(t)} \circ\left(\varphi \otimes \varphi \circ P^{(t)}\right)\right)\left(P^{(s)} x\right)
$$

где

$$
\left(P^{(t)} \circ\left(\varphi \otimes \varphi \circ P^{(s)}\right)\right)(a \otimes b):=\varphi \otimes \varphi\left(P^{(s)} b\right) P^{(t)} a, \quad a, b \in \mathfrak{A} .
$$

Будем говорить, что к.к.с.п. $\left(P^{(t)}, \varphi\right)$ является стационарным, если для любого $t \geqslant 1$

$$
\varphi \otimes \varphi\left(P^{(t)} x\right)=\varphi(x) \quad \forall x \in \mathfrak{A} .
$$

Это условие естественно возникает при изучении моделей наследственности, согласующихся с законами Менделя (см. [1], [4], [9]). Поэтому далее (в $\S 11$ и 2) мы всегда будем считать, что к.к.с.п. является стационарным.

Если $C^{*}$-алгебра $\mathfrak{A}$ совпадает с конечномерной коммутативной алгеброй, т.е. $\mathfrak{A}=\left\{\mathbf{x} \in \mathbb{R}^{n}:\|\mathbf{x}\|=\max _{1 \leqslant i \leqslant n}\left|x_{i}\right|, \mathbf{x}=\left(x_{i}\right)_{i=1}^{n}\right\}$, то к.к.с.п. $\left(P^{(t)}, \varphi\right)$ совпадает с однородным квадратичным стохастическим процессом $\left(p_{i j, k}^{(t)}\right)_{i, j, k=1}^{n}$ типа $A$, определенньм в [10]. Действительно, полагая

$$
p_{i j, k}^{(t)}=\left(P^{(t)} e_{k}\right)_{i j}
$$

где $e_{k}=(0,0, \ldots, 0, \underset{k}{1}, 0, \ldots, 0), i, j, k=\overline{1, n}$ и $\varphi=\left\{m_{i}\right\}_{i=1}^{n}, m_{i} \geqslant 0, \sum_{i=1}^{n} m_{i}=1$, легко видеть, что справедливы все условия, налагаемые на квадратичньй стохастический процесс, т.е.

$$
p_{i j, k}^{(t)} \geqslant 0, \quad p_{i j, k}^{(t)}=p_{j i, k}^{(t)}, \quad \sum_{k=1}^{n} p_{i j, k}^{(t)}=1,
$$

и при этом уравнение (1.1) принимает вид

$$
p_{i j, k}^{(t+s)}=\sum_{m, l=1}^{n} \sum_{q, r=1}^{n} p_{i j, l}^{(t)} p_{q r, m}^{(t)} p_{l m, k}^{(s)} m_{q} m_{r} .
$$

Обратно, если мы имеем квадратичный стохастический процесс $\left(p_{i j, k}^{(t)}\right)_{i, j, k=1}^{n}$ с начальным состоянием $\left\{m_{i}\right\}_{i=1}^{n}$, удовлетворяющий $(*)$ и $(* *)$, то мы можем определить к.к.с.п. на $l_{n}^{\infty}=\left\{\mathbf{x} \in \mathbb{R}^{n}:\|\mathbf{x}\|=\max _{1 \leqslant i \leqslant n}\left|x_{i}\right|, \mathbf{x}=\left(x_{i}\right)_{i=1}^{n}\right\}$ следующим образом:

$$
\begin{aligned}
\left(P^{(t)} f\right)_{i j} & =\sum_{k=1}^{n} f_{k} p_{i j, k}^{(t)}, \quad i, j=\overline{1, n}, \\
\varphi(f) & =\sum_{k=1}^{n} f_{k} m_{k}, \quad f=\left(f_{i}\right)_{i=1}^{n} \in l_{n}^{\infty} .
\end{aligned}
$$


Таким образом, определение 1.1 обобщает ранее известное понятие квадратичного стохастического процесса (к.с.п.), изучению которого посвящены работы [10]-[12]. В этих работах показано, что такие процессы возникают при описании некоторых физических, химических, биологических процессов, и обоснована необходимость условия $t \geqslant 1$ в определении к.с.п.

Приведем пример стационарного к.к.с.п. Для этого напомним, что линейный оператор $T: \mathfrak{A} \rightarrow \mathfrak{A}$ называется марковским, если он удовлетворяет условиям $T\left(\mathfrak{A}_{+}\right) \subset \mathfrak{A}_{+}$и $T 1_{\mathfrak{A}}=1_{\mathfrak{A}}$.

Состояние $\varphi \in S$ называется инвариантным относительно $T$, если $\varphi(T x)=$ $\varphi(x)$ для всех $x \in \mathfrak{A}$.

Пусть $T^{t}$ - марковская полугруппа операторов на $\mathfrak{A}$ с инвариантњым состоянием $\varphi$, т.е. $\varphi \circ T^{t}=\varphi$ для всех $t \in \mathbb{R}_{+}$. Определим семейство $H^{(t)}$ следующим образом:

где

$$
H^{(t)} x=\frac{1}{2}\left(T^{(t)} x \otimes 1+1 \otimes T^{(t)} x\right), \quad x \in \mathfrak{A}
$$

$$
T^{(t)} x=\frac{1}{2^{t-1}}\left(T^{t} x+\left(2^{t-1}-1\right) \varphi(x)\right) .
$$

Тогда легко проверить, что $\left(H^{(t)}, \varphi \otimes \varphi\right)$ является стационарным к.к.с.п. на $\mathfrak{A}$.

Состояние $\nu \in S$ называется инвариантным относительно к.к.с.п. $\left(P^{(t)}, \varphi\right)$, если выполнено равенство $\nu \otimes \varphi \circ P^{(t)}=\nu$ при всех $t \geqslant 1$.

ОПРЕДЕЛЕНИЕ 1.2. Будем говорить, что к.к.с.п. $\left(P^{(t)}, \varphi\right)$ является строго эргодическим, если он имеет единственное инвариантное состояние $\varphi$, т.е. из равенства $\nu \otimes \varphi \circ P^{(t)}=\nu$ при всех $t \geqslant 1$ следует $\nu=\varphi$.

Далее вместо $\left(P^{(t)}, \varphi\right)$ будем использовать просто символ $P^{(t)}$ и будем предполагать, что для любого $x \in \mathfrak{A}$ функция $\left\|P^{(t)} x\right\|$ измерима (в смысле Лебега) на $\mathbb{R}_{+}$.

Лемма 1.1. Пусть $h \in \mathfrak{A}^{*}$ - инвариантный функционал для строго әргодического к.к.с.п. $P^{(t)}$. Тогда справедливо равенство

$$
h=\lambda \varphi, \quad \lambda \in \mathbb{C} .
$$

ДокАЗАТЕЛЬство. Предположим сперва, что $h$ - положительный функционал. Тогда $\psi=h / h\left(1_{\mathfrak{A}}\right)$ является состоянием. Так как к.к.с.п. $P^{(t)}$ строго эргодичен, то имеем $h / h\left(1_{\mathfrak{A}}\right)=\varphi$, откуда $h=h\left(1_{\mathfrak{A}}\right) \varphi$.

Пусть теперь $h$ эрмитов, т.е. $h\left(x^{*}\right)=\overline{h(x)}$, тогда для него сушествует единственное разложение (см. [8; теорема 1.14.3]) такое, что

$$
h=h_{+}-h_{-}, \quad\|h\|_{1}=\left\|h_{+}\right\|_{1}+\left\|h_{-}\right\|_{1},
$$

где $\|\cdot\|_{1}-$ норма на $\mathfrak{A}^{*}$. Отсюда для каждого $t \geqslant 1$

$$
h \otimes \varphi \circ P^{(t)}=h_{+} \otimes \varphi \circ P^{(t)}-h_{-} \otimes \varphi \circ P^{(t)}=h_{+}-h_{-} .
$$

Так как $\left\|h_{+} \otimes \varphi \circ P^{(t)}\right\|_{1}=h_{+}\left(1_{\mathfrak{A}}\right),\left\|h_{-} \otimes \varphi \circ P^{(t)}\right\|_{1}=h_{-}\left(1_{\mathfrak{A}}\right)$, то из единственности разложения (1.2) находим $h_{+} \otimes \varphi \circ P^{(t)}=h_{+}, h_{-} \otimes \varphi \circ P^{(t)}=h_{-}$. Из доказанного вьше следует $h=\lambda \varphi$.

Пусть $h-$ произвольный функционал. Тогда для него существуют эрмитовы функционалы $h_{1}, h_{2}$ такие, что $h=h_{1}+i h_{2}$. В силу инвариантности $h$ имеем

$$
h_{1} \otimes \varphi \circ P^{(t)}+i h_{2} \otimes \varphi \circ P^{(t)}=h_{1}+i h_{2},
$$

отсюда находим $h_{j} \otimes \varphi \circ P^{(t)}=h_{j}, j=1,2$. Тем самым получаем $h_{j}=\lambda_{j} \varphi$, $j=1,2$. Таким образом, $h=\left(\lambda_{1}+i \lambda_{2}\right) \varphi$. Лемма доказана. 
Теорема 1.1. Пусть $P^{(t)}$ - к.к.c.n. на $C^{*}$-алгебре $\mathfrak{A}$ с единицей. Тогда следующие условия әквивалентны:

(i) K.к.c.n. $P^{(t)}$ является строго эргодическим;

(ii) для любого $x \in \mathfrak{A}$ имеет место

$$
\lim _{T \rightarrow \infty} \frac{1}{T} \int_{1}^{T} P^{(t)} x d t=\varphi(x) 1_{\mathfrak{A} \otimes \mathfrak{A}},
$$

где сходимость равномерная, т.е. по норме;

(iii) для кажсдого состояния $\psi \in S^{2}$ u $x \in \mathfrak{A}$ имеет место

$$
\lim _{T \rightarrow \infty} \frac{1}{T} \int_{1}^{T} \psi\left(P^{(t)} x\right) d t=\varphi(x) .
$$

ДокАЗАТЕЛЬство. (i) $\Rightarrow$ (ii). Рассмотрим элементы вида

$$
y=\varphi \circ P^{(\tau)} x-x, \quad x \in \mathfrak{A}, \quad \tau \geqslant 1 .
$$

Для таких $y$ в силу (1.1) имеем

$$
P^{(t)} y=\left(P^{(t)} \circ \varphi\right)\left(P^{(\tau)} x\right)-P^{(t)} x=P^{(t+\tau)} x-P^{(t)} x, \quad t \geqslant 1,
$$

откуда получаем

$$
\begin{aligned}
\frac{1}{T} \int_{1}^{T} P^{(t)} y d t & =\frac{1}{T} \int_{1}^{T}\left(P^{(t+\tau)} x-P^{(t)} x\right) d t \\
& =\frac{1}{T}\left(\int_{T}^{T+\tau} P^{(t)} x d t-\int_{1}^{1+\tau} P^{(t)} x d t\right)
\end{aligned}
$$

Из этого вытекает, что

$$
\left\|\frac{1}{T} \int_{1}^{T} P^{(t)} y d t\right\| \leqslant \frac{2 \tau}{T}\|x\| \rightarrow 0, \quad T \rightarrow \infty .
$$

При этом $\varphi(y)=\varphi \otimes \varphi\left(P^{(\tau)} x\right)-\varphi(x)=\varphi(x)-\varphi(x)=0$. Итак, имеем

$$
\frac{1}{T} \int_{1}^{T} P^{(t)} y d t \rightarrow \varphi(y) 1_{\mathfrak{A} \otimes \mathfrak{A}} \quad \text { при } \quad T \rightarrow \infty .
$$

Замыкание линейной оболочки элементов вида (1.3) обозначим через $\mathfrak{B}$, а через $\mathfrak{B}_{0}$ обозначим ядро состояния $\varphi$, т.е. $\mathfrak{B}_{0}=\{x \in \mathfrak{A}: \varphi(x)=0\}$. Ясно, что $\mathfrak{B} \subseteq \mathfrak{B}_{0}$. Покажем, что $\mathfrak{B}=\mathfrak{B}_{0}$. Предположим противное: $\mathfrak{B} \neq \mathfrak{B}_{0}$, т.е. найдется элемент $x_{0} \in \mathfrak{B}_{0}$ такой, что $x_{0} \notin \mathfrak{B}$. По теореме Хана-Банаха сушествует такой непрерьвньй функционал $h$ на $\mathfrak{A}$, что $\left.h\right|_{\mathfrak{B}}=0$ и $h\left(x_{0}\right)=1$. Легко видеть, что $h \otimes \varphi \circ P^{(t)}=h$. Тогда в силу леммы 1.1 имеем $h=\lambda \varphi$ в противоречии с $\varphi\left(x_{0}\right)=0$. Следовательно, $\mathfrak{B}=\mathfrak{B}_{0}$.

Пусть $y \in \mathfrak{B}_{0}$. Тогда для заданного $\varepsilon>0$ найдем элемент $y_{\varepsilon}=\varphi \circ P^{\left(\tau_{\varepsilon}\right)} x_{\varepsilon}-x_{\varepsilon}$ такой, что $\left\|y-y_{\varepsilon}\right\|<\varepsilon / 2$. В силу условия

$$
\frac{1}{T} \int_{1}^{T} P^{(t)} y_{\varepsilon} d t \rightarrow 0 \text { при } T \rightarrow \infty
$$


выберем $T_{0}$ так, что $\left\|\frac{1}{T} \int_{1}^{T} P^{(t)} y_{\varepsilon} d t\right\|<\varepsilon / 2$ при всех $T \geqslant T_{0}$. Тогда получим

$$
\begin{aligned}
\left\|\frac{1}{T} \int_{1}^{T} P^{(t)} y d t\right\| & \leqslant\left\|\frac{1}{T} \int_{1}^{T} P^{(t)}\left(y-y_{\varepsilon}\right) d t\right\|+\left\|\frac{1}{T} \int_{1}^{T} P^{(t)} y_{\varepsilon} d t\right\| \\
& \leqslant\left\|y-y_{\varepsilon}\right\|+\frac{\varepsilon}{2}<\varepsilon \text { при всех } T \geqslant T_{0} .
\end{aligned}
$$

Итак, имеем

$$
\lim _{T \rightarrow \infty} \frac{1}{T} \int_{1}^{T} P^{(t)} y d t=0=\varphi(y) 1_{\mathfrak{A} \otimes \mathfrak{A}} \quad \text { для всех } \quad y \in \mathfrak{B}_{0} .
$$

Пусть $x \in \mathfrak{A}$. Положим $y=\varphi(x) 1_{\mathfrak{A}}-x$. Очевидно, $y \in \mathfrak{B}_{0}$ и, следовательно, для $y$ имеет место последнее соотношение, откуда

$$
\lim _{T \rightarrow \infty} \frac{1}{T} \int_{1}^{T} P^{(t)} x d t=\varphi(x) 1_{\mathfrak{A} \otimes \mathfrak{A}}
$$

Импликация (ii) $\Rightarrow$ (iii) очевидна.

(iii) $\Rightarrow($ i $)$. Пусть $\nu$-инвариантное состояние для к.к.с.п. $P^{(t)}$. Из непрерывности $\nu \otimes \varphi \in S^{2}$ следует, что для любого $x \in \mathfrak{A}$

$$
\nu \otimes \varphi\left(\frac{1}{T} \int_{1}^{T} P^{(t)} x d t\right) \rightarrow \varphi(x) \text { при } T \rightarrow \infty
$$

С другой стороны,

$$
\nu \otimes \varphi\left(\frac{1}{T} \int_{1}^{T} P^{(t)} x d t\right)=\frac{1}{T} \int_{1}^{T} \nu \otimes \varphi\left(P^{(t)} x\right) d t=\nu(x), \quad x \in \mathfrak{A}
$$

Таким образом, $\nu=\varphi$. Теорема доказана.

Заметим, что аналогичная теорема была доказана для автоморфизма компактного метрического пространства в [13; гл. $1, \S 7$, теорема 2].

\section{§2. Равномерная эргодическая теорема с весом}

Этот параграф̆ посвяшен изучению сходимости в равномерной топологии весовых средних вида

$$
\left\{\frac{1}{T} \int_{1}^{T} b(t) P^{(t)} x d t\right\}, \quad \text { где } b(t) \in \mathbb{C}, \quad t \geqslant 1 .
$$

Рассмотрим стационарньй к.к.с.п. $P^{(t)}$ на $C^{*}$-алгебре $\mathfrak{A}$ с единицей. Пусть $K=\{z \in \mathbb{C}:|z|=1\}$, через $\mu$ обозначим нормированную меру Хаара на $K$ и $(Z, \lambda)=\times_{i=1}^{s}(K, \mu)$ для некоторого $s \in \mathbb{N}$. Положим $\mathfrak{M}=C(Z ; \mathfrak{A})-$ совокупность всех $\mathfrak{A}$-значных непрерывных функций на $Z$. Определим семейства линейных отображений $P_{\alpha}^{(t)}: C(Z) \rightarrow C(Z) \otimes C(Z), Q_{\alpha}^{(t)}: \mathfrak{M} \rightarrow \mathfrak{M} \otimes \mathfrak{M}$, где $C(Z)$-пространство 
всех непрерывных функций на $Z$, и линейный функционал $\varphi \otimes \lambda: \mathfrak{M} \rightarrow \mathbb{C}$ следующим образом:

$$
\begin{aligned}
\left(P_{\alpha}^{(t)} f\right)(\mathbf{x}, \mathbf{y}) & =\frac{1}{2}\left(\left(T_{\alpha}^{(t)} f\right)(\mathbf{x})+\left(T_{\alpha}^{(t)} f\right)(\mathbf{y})\right) \\
Q_{\alpha}^{(t)}(x \otimes f) & =P^{(t)} x \otimes P_{\alpha}^{(t)} f, \quad x \in \mathfrak{A}, \quad f \in C(Z), \\
\varphi \otimes \lambda(f) & =\int_{Z} \varphi(f(\mathbf{x})) d \lambda(\mathbf{x})
\end{aligned}
$$

где

$$
\left(T_{\alpha}^{(t)} f\right)(\mathbf{x})=\frac{1}{2^{t-1}}\left(f\left(\alpha^{t} \mathbf{x}\right)+\left(2^{t-1}-1\right) \lambda(f)\right),
$$

$\mathbf{x}=\left(x_{1}, \ldots, x_{s}\right), \mathbf{y}=\left(y_{1}, \ldots, y_{s}\right) \in Z, \alpha=\left\{\alpha_{i}\right\}_{i=1}^{s} \subset K$. Легко видеть, что определенное семейство $\left(Q_{\alpha}^{(t)}, \varphi \otimes \lambda\right)$ является стационарным к.к.с.п., т.е. $(\varphi \otimes \lambda) \otimes$ $(\varphi \otimes \lambda) \circ Q_{\alpha}^{(t)}=\varphi \otimes \lambda$.

ОПРЕДЕЛЕНИЕ 2.1. К.к.с.П. $P^{(t)}$ называется строго перемешивающим, если для любого $x \in \mathfrak{A}$ имеет место соотношение

$$
\lim _{T \rightarrow \infty} \frac{1}{T} \int_{1}^{T}\left\|P^{(t)} x-\varphi(x) 1_{\mathfrak{A} \otimes \mathfrak{A}}\right\| d t=0 .
$$

Теорема 2.1. Пусть $P^{(t)}$ - к.к.c.n. на $\mathfrak{A}$. Тогда следующие условия әквивалентны:

(i) $P^{(t)}$ - строго перемешивающии;

(ii) к.к.c.n. $\left(Q^{(t)}, \varphi \otimes \varphi\right)$ на $\mathfrak{A} \otimes \mathfrak{A}$, определенный на әлементах вида $x \otimes y$ как

$$
Q^{(t)}(x \otimes y)=\frac{1}{2}\left(P^{(t)} x \otimes P^{(t)} y+P^{(t)} y \otimes P^{(t)} x\right),
$$

строго эргодичен.

ДокАЗАтЕльство. (i) $\Rightarrow\left(\right.$ ii). Пусть $P^{(t)}$ - строго перемешиваюший к.к.с.п. Пусть $x, y \in \operatorname{ker} \varphi$, тогда

$$
\begin{aligned}
& \lim _{T \rightarrow \infty} \frac{1}{T} \int_{1}^{T}\left\|P^{(t)} x\right\| d t=0 \\
& \lim _{T \rightarrow \infty} \frac{1}{T} \int_{1}^{T}\left\|P^{(t)} y\right\| d t=0 .
\end{aligned}
$$

Мы воспользуемся следующим простьм утверждением, касающимся числовых функций на $\left(\mathbb{R}_{+}, \mathfrak{S}, m\right)$, где $\mathfrak{S}-\sigma$-алгебра измеримых по Лебегу множеств, а $m$ мера Лебега: для любой ограниченной измеримой функиии $f(x)$ соотношение

$$
\lim _{T \rightarrow \infty} \frac{1}{T} \int_{1}^{T}|f(t)| d m(t)=0
$$

әквивалентно тому, что найдется измеримое множество $A$ в $\mathbb{R}_{+}$nлотности нуль, т.е. $\lim _{T \rightarrow \infty} \frac{1}{T} m(A \cap[1, T])=0$, такое, что

$$
\lim _{\substack{x \rightarrow \infty \\ x \notin A}}|f(x)|=0 .
$$


Из этого утверждения вытекает, что для функций $\left\|P^{(t)} x\right\|,\left\|P^{(t)} y\right\|, t \geqslant 1$, найдутся исключительные измеримые множества $A_{x}$ и $A_{y}$ плотности нуль такие, что $\left\|P^{(t)} x\right\|$ и $\left\|P^{(t)} y\right\|$ стремятся к нулю, когда $t \rightarrow \infty$ вне соответствуюшего исключительного множества $A_{x}$ и $A_{y}$. Множество $A=A_{x} \cup A_{y}$ также имеет плотность нуль, и если $t \rightarrow \infty$ вне $A$, то $\left\|P^{(t)} x\right\| \cdot\left\|P^{(t)} y\right\|$ стремится к нулю. Снова пользуясь сформулированным утверждением, получаем, что

$$
\lim _{T \rightarrow \infty} \frac{1}{T} \int_{1}^{T}\left\|P^{(t)} x\right\| \cdot\left\|P^{(t)} y\right\| d t=0
$$

Отсюда следует, что

$$
\left\|\frac{1}{T} \int_{1}^{T} Q^{(t)}(x \otimes y) d t\right\| \leqslant \frac{1}{T} \int_{1}^{T}\left\|P^{(t)} x\right\| \cdot\left\|P^{(t)} y\right\| d t \rightarrow 0 \text { при } T \rightarrow \infty .
$$

В обшем случае для $x, y \in \mathfrak{A}$ положим $x^{0}=\varphi(x) 1_{\mathfrak{A}}-x, y^{0}=\varphi(y) 1_{\mathfrak{A}}-y$. Ясно, что $x^{0}, y^{0} \in \operatorname{ker} \varphi$, тогда из полученного выше и строгой перемешиваемости к.к.с.п. $P^{(t)}$ имеем

$$
\frac{1}{T} \int_{1}^{T} Q^{(t)}(x \otimes y) d t \rightarrow \varphi(x) \varphi(y) 1_{(\mathfrak{A} \otimes \mathfrak{A}) \otimes(\mathfrak{A} \otimes \mathfrak{A})} \quad \text { при } \quad T \rightarrow \infty .
$$

Пусть $z \in \mathfrak{A} \otimes \mathfrak{A}$, тогда для произвольного $\varepsilon>0$ сушествует элемент $\sum_{i=1}^{m} x_{i} \otimes y_{i}$ такой, что $\left\|z-\sum_{i=1}^{m} x_{i} \otimes y_{i}\right\|<\varepsilon$. Из (2.1) следует, что сушествует такое $T_{0} \in \mathbb{R}_{+}$, что

$$
\left\|\frac{1}{T} \int_{1}^{T} Q^{(t)}\left(\sum_{i=1}^{m} x_{i} \otimes y_{i}\right) d t-\varphi \otimes \varphi\left(\sum_{i=1}^{m} x_{i} \otimes y_{i}\right) 1_{(\mathfrak{A} \otimes \mathfrak{A}) \otimes(\mathfrak{A} \otimes \mathfrak{A})}\right\|<\varepsilon
$$

при всех $T \geqslant T_{0}$. Тогда стандартным образом получим

$$
\left\|\frac{1}{T} \int_{1}^{T} Q^{(t)} z d t-\varphi \otimes \varphi(z) 1_{(\mathfrak{A} \otimes \mathfrak{A}) \otimes(\mathfrak{A} \otimes \mathfrak{A})}\right\| \leqslant 3 \varepsilon \quad \text { при всех } T \geqslant T_{0} .
$$

Используя теорему 1.1, получаем строгую эргодичность $Q^{(t)}$.

(ii) $\Rightarrow(\mathrm{i})$. Пусть к.к.с.п. $Q^{(t)}$ строго эргодичен и $x \in \operatorname{ker} \varphi, x=x^{*}$. Тогда для произвольного $\varepsilon>0$ сушествует $T_{0, x} \in \mathbb{R}_{+}$такое, что

$$
\left\|\frac{1}{T} \int_{1}^{T} P^{(t)} x \otimes P^{(t)} x d t\right\|<\varepsilon \text { при всех } T \geqslant T_{0, x},
$$

и для произвольного эрмитова $\psi \in(\mathfrak{A} \otimes \mathfrak{A})_{1}^{*}=\left\{\nu \in(\mathfrak{A} \otimes \mathfrak{A})^{*}:\|\nu\|_{1} \leqslant 1\right\}$ имеем

$$
\left|\frac{1}{T} \int_{1}^{T} \psi \otimes \psi\left(P^{(t)} x \otimes P^{(t)} x\right) d t\right|<\varepsilon \quad \text { при всех } T \geqslant T_{0, x} .
$$

Отсюда в силу самосопряженности элемента $x$ получим

$$
\frac{1}{T} \int_{1}^{T}\left|\psi\left(P^{(t)} x\right)\right|^{2} d t<\varepsilon \text { при всех } T \geqslant T_{0, x},
$$


так что

$$
\frac{1}{T} \int_{1}^{T}\left|\psi\left(P^{(t)} x\right)\right| d t<\varepsilon \quad \text { при всех } T \geqslant T_{0, x} \quad \text { и } \quad \psi \in(\mathfrak{A} \otimes \mathfrak{A})_{1, h}^{*},
$$

где $(\mathfrak{A} \otimes \mathfrak{A})_{1, h}^{*}-$ эрмитова часть $(\mathfrak{A} \otimes \mathfrak{A})_{1}^{*}$. Итак, имеем

$$
\frac{1}{T} \int_{1}^{T} \sup _{\psi \in(\mathfrak{A} \otimes \mathfrak{A})_{1, h}^{*}}\left|\psi\left(P^{(t)} x\right)\right| d t<\varepsilon \quad \text { при всех } T \geqslant T_{0, x} .
$$

Введем следующий функционал

$$
\|x\|_{*}=\sup \left\{|\psi(x)|: \psi \in(\mathfrak{A} \otimes \mathfrak{A})_{1, h}^{*}\right\}, \quad x \in \mathfrak{A} \otimes \mathfrak{A} .
$$

Докажем одну вспомогательную лемму.

ЛЕмма 2.1. Функционал $\|\cdot\|_{*}$ является нормой на $\mathfrak{A} \otimes \mathfrak{A}$, и эта норма әквивалентна норме $\|\cdot\|$, т.е. имеет место неравенство

$$
\|x\|_{*} \leqslant\|x\| \leqslant 2\|x\|_{*} \quad \forall x \in \mathfrak{A} \otimes \mathfrak{A} .
$$

ДокАЗАтЕЛьство. Ясно, что функционал $\|\cdot\|_{*}$ положителен. Пусть для некоторого $x \in \mathfrak{A} \otimes \mathfrak{A}\|x\|_{*}=0$. Отсюда следует, что $\varphi(x)=0$ для всех $\varphi \in(\mathfrak{A} \otimes \mathfrak{A})_{1, h}^{*}$. Так как каждый элемент $\psi \in(\mathfrak{A} \otimes \mathfrak{A})_{1}^{*}$ представим в виде $\psi=\varphi_{1}+i \varphi_{2}, \varphi_{1}, \varphi_{2} \in(\mathfrak{A} \otimes \mathfrak{A})_{1, h}^{*}$, то получим $\psi(x)=0$. В силу произвольности $\psi$ имеем $x=0$. Остальные условия нормы очевидны. Для доказательства эквивалентности норм заметим, что

$$
\|x\|=\sup \left\{|\psi(x)|: \psi \in(\mathfrak{A} \otimes \mathfrak{A})_{1}^{*}\right\} .
$$

Отсюда следует, что

$$
\|x\|_{*} \leqslant\|x\| \quad \forall x \in \mathfrak{A} \otimes \mathfrak{A} .
$$

Как уже было сказано, каждый функционал $\psi \in(\mathfrak{A} \otimes \mathfrak{A})_{1}^{*}$ представляется в виде $\psi=\varphi_{1}+i \varphi_{2}, \varphi_{1}, \varphi_{2} \in(\mathfrak{A} \otimes \mathfrak{A})_{1, h}^{*}$. Отсюда имеем

$$
\|x\| \leqslant \sup \left\{\left|\varphi_{1}(x)\right|: \varphi_{1} \in(\mathfrak{A} \otimes \mathfrak{A})_{1, h}^{*}\right\}+\sup \left\{\left|\varphi_{2}(x)\right|: \varphi_{2} \in(\mathfrak{A} \otimes \mathfrak{A})_{1, h}^{*}\right\}=2\|x\|_{*} .
$$

Лемма доказана.

Продолжим доказательство теоремы 2.1. Итак, из (2.2) получим

$$
\frac{1}{T} \int_{1}^{T}\left\|P^{(t)} x\right\|_{*} d t<\varepsilon \text { при всех } T \geqslant T_{0, x},
$$

откуда в силу леммы 2.1 имеем

$$
\frac{1}{T} \int_{1}^{T}\left\|P^{(t)} x\right\| d t<\frac{\varepsilon}{2} \text { при всех } T \geqslant T_{0, x} .
$$

Пусть теперь $x \in \operatorname{ker} \varphi-$ произвольньй элемент. Тогда $x$ представляется в виде $x=x_{1}+i x_{2}$, где $x_{j}=x_{j}^{*}, x_{j} \in \operatorname{ker} \varphi, j=1,2$, и из (2.3) имеем

$$
\frac{1}{T} \int_{1}^{T}\left\|P^{(t)} x\right\| d t<\varepsilon \text { при всех } T \geqslant \max \left\{T_{0, x_{1}}, T_{0, x_{2}}\right\} .
$$

Таким образом, из (2.4) легко следует строгая перемешиваемость к.к.с.п. $P^{(t)}$. Теорема доказана.

Аналогичньми рассуждениями мы можем доказать следующую теорему. 
Теорема 2.2. Пусть $P^{(t)}$ и $P_{\alpha}^{(t)}$ - строго перемешивающие к.к.c.n. Тогда к.к.с.п. $Q_{\alpha}^{(t)}$ строго эргодичен.

ЛЕмма 2.2. Пусть $T$ - марковский оператор с инвариантным состоянием. . Если выполнено равенство

$$
\frac{1}{2}(\nu \circ T+\varphi)=\nu
$$

то имеет место $\nu=\varphi$.

ДокАЗАТЕльство. Пусть $x \in \operatorname{ker} \varphi$, т.е. $\varphi(x)=0$. Тогда

$$
\nu(T x)=2 \nu(x)
$$

Не ограничивая общности, можно предполагать, что $\|x\| \leqslant 1$. Тогда $|\nu(T x)| \leqslant 1$ и $|\nu(x)| \leqslant 1 / 2$. Из $\varphi \circ T=\varphi$ следует $T x \in \operatorname{ker} \varphi$, откуда $|\nu(T x)| \leqslant 1 / 2$. Из (2.6) имеем $|\nu(x)| \leqslant 1 / 2^{2}$. Продолжая этот процесс для любого $n \in \mathbb{N}$, получаем $|\nu(x)| \leqslant 1 / 2^{n}$, что означает $\nu(x)=0$. Отсюда заключаем, что $\operatorname{ker} \varphi \subset \operatorname{ker} \nu$.

Пусть $x \in \operatorname{ker} \nu$. Тогда $\nu(T x)=-\varphi(x)$. Предположим, что $x \notin \operatorname{ker} \varphi$, что означает $\nu(T x) \neq 0$. Из $(2.5)$ получаем

$$
\begin{gathered}
\nu(T x)+\varphi(x)=2 \nu(x), \\
\nu\left(T^{2} x\right)+\varphi(x)=2 \nu(T x),
\end{gathered}
$$

отсюда находим

$$
\nu\left(T^{2} x\right)-\nu(T x)=2(\nu(T x)-\nu(x)) .
$$

Аналогичным образом для любого $x \in \mathbb{N}$ получаем

$$
\nu\left(T^{n+1} x\right)-\nu\left(T^{n} x\right)=2\left(\nu\left(T^{n} x\right)-\nu\left(T^{n-1} x\right)\right) .
$$

Из последнего равенства заключаем

$$
\begin{aligned}
\nu\left(T^{n+1} x\right)-\nu\left(T^{n} x\right) & =2^{n}(\nu(T x)-\nu(x)), \\
\nu\left(T^{n+1} x\right) & =\nu\left(T^{n} x\right)+2^{n} \nu(T x),
\end{aligned}
$$

здесь мы воспользовались тем, что $\nu(x)=0$. В силу $\|x\| \leqslant 1$ имеем $\left|\nu\left(T^{n+1} x\right)\right| \leqslant 1$. Тогда в силу (2.7) получаем $\nu(T x)=0$, что противоречит нашему предположению. Тем самым, $\operatorname{ker} \nu \subset \operatorname{ker} \varphi$. Из доказанного вьше следует, что $\operatorname{ker} \varphi=\operatorname{ker} \nu$.

Пусть $x \in \mathfrak{A}$ - произвольный элемент из $\mathfrak{A}$. Тогда элемент $y=\varphi(x) 1_{\mathfrak{A}}-x$ принадлежит $\operatorname{ker} \varphi$, т.е. $\varphi(y)=0$. Из доказанного выше равенства следует $\nu(y)=0$, что означает $\nu(x)=\varphi(x)$. Лемма доказана.

Приведем пример строго перемешивающего к.к.с.п.

Рассмотрим на $C^{*}$-алгебре $\mathfrak{A}$ с единицей 1 к.к.с.п. $H^{(t)}$, определенный в $\S 1$. На $\mathfrak{A} \otimes \mathfrak{A}$ определим к.к.с.п. $Q^{(t)}$ как в теореме 2.1 , т.е.

$$
Q^{(t)}(x \otimes y)=\frac{1}{2}\left(H^{(t)} x \otimes H^{(t)} y+H^{(t)} y \otimes H^{(t)} x\right), \quad x, y \in \mathfrak{A} .
$$

Тогда легко проверить, что для состояния $\widetilde{\varphi}=\varphi \otimes \varphi$ справедливо

$$
\widetilde{\varphi} \otimes \widetilde{\varphi} \circ Q^{(t)}=\widetilde{\varphi} \text { при } t \geqslant 1 \text {. }
$$


Покажем, что определенный к.к.с.п. $\left(Q^{(t)}, \widetilde{\varphi}\right)$ строго эргодичен. В самом деле, пусть $\widetilde{\psi} \in S^{2}$ - инвариантное состояние для $Q^{(t)}$, т.е.

$$
\widetilde{\psi} \otimes \widetilde{\varphi} \circ Q^{(t)}=\widetilde{\psi}
$$

Согласно определениям $H^{(t)}$ и $Q^{(t)}$ последнее равенство перепишется как

$$
\frac{1}{2}\left(\frac{\widetilde{\psi}\left(T^{t} x \otimes 1\right)+\widetilde{\psi}\left(1 \otimes T^{t} x\right)}{2} \varphi(y)+\frac{\widetilde{\psi}\left(T^{t} y \otimes 1\right)+\widetilde{\psi}\left(1 \otimes T^{t} y\right)}{2} \varphi(x)\right)=\widetilde{\psi}(x \otimes y),
$$

где $x, y \in \mathfrak{A}$.

Из (2.8), полагая по отдельности $x=1$ и $y=1$, получаем равенства

$$
\begin{aligned}
& \frac{1}{2}\left(\varphi(y)+\frac{\widetilde{\psi}\left(T^{t} y \otimes 1\right)+\widetilde{\psi}\left(1 \otimes T^{t} y\right)}{2}\right)=\widetilde{\psi}(1 \otimes y), \\
& \frac{1}{2}\left(\frac{\widetilde{\psi}\left(T^{t} x \otimes 1\right)+\widetilde{\psi}\left(1 \otimes T^{t} x\right)}{2}+\varphi(x)\right)=\widetilde{\psi}(x \otimes 1) .
\end{aligned}
$$

Из последнего следует

$$
\widetilde{\psi}(x \otimes 1)=\widetilde{\psi}(1 \otimes x), \quad x \in \mathfrak{A} .
$$

Отсюда и из (2.9) находим

$$
\frac{1}{2}\left(\widetilde{\psi}\left(T^{t} x \otimes 1\right)+\varphi(x)\right)=\widetilde{\psi}(x \otimes 1) .
$$

Теперь, полагая $\psi(x)=\widetilde{\psi}(x \otimes 1)$ и применяя лемму 2.2, получаем

$$
\psi=\varphi
$$

и из (2.8), (2.10) вытекает

$$
\widetilde{\psi}(x \otimes y)=\varphi(x) \varphi(y) \text { для всех } x, y \in \mathfrak{A},
$$

что означает строгую эргодичность $Q^{(t)}$. В силу теоремы 2.1 получим строгую перемешиваемость $H^{(t)}$.

Через $F$ обозначим совокупность всех наборов $\left\{\alpha_{i}\right\}_{i=1}^{s}$ таких, что к.к.с.п. $P_{\alpha}^{(t)}$ - строго перемешивающий, т.е.

$$
F=\left\{\alpha=\left\{\alpha_{i}\right\}_{i=1}^{s}: P_{\alpha}^{(t)}-\text { строго перемешивающий, } \alpha_{i} \in K, s \in \mathbb{N}\right\} .
$$

Напомним, что ограниченная последовательность $\left\{b_{n}\right\} \subset \mathbb{C}$ называется последовательностью Безиковича, если для произвольного $\varepsilon>0$ существует тригонометрический полином

$$
W_{\varepsilon}(j)=\sum_{s} y_{s} \exp \left(i \theta_{s} j\right)
$$

такой, что

$$
\varlimsup_{n \rightarrow \infty} \frac{1}{n} \sum_{j=1}^{n}\left|b_{j}-W_{\varepsilon}(j)\right|<\varepsilon .
$$

По аналогии с этим определением введем следующее 
ОПРЕДЕЛЕНИЕ 2.2. Ограниченная измеримая функция $b(t)$, определенная при $t \geqslant 1$, назьвается $\Phi$-функцией Безиковича, если для любого $\varepsilon>0$ существует набор $\alpha=\left\{\alpha_{i}\right\}_{i=1}^{s} \in F$, функция $f \in C(Z)$ и $\mathbf{x}, \mathbf{y} \in Z$ такие, что

$$
\varlimsup_{T \rightarrow \infty} \frac{1}{T} \int_{1}^{T}\left|b(t)-\left(P_{\alpha}^{(t)} f\right)(\mathbf{x}, \mathbf{y})\right| d t<\varepsilon
$$

Пусть $P^{(t)}$ - строго перемешиваюший к.к.с.п. на $C^{*}$-алгебре $\mathfrak{A}$ и $\alpha=\left\{\alpha_{i}\right\}_{i=1}^{s}$ $\in F$. Тогда из теоремы 2.2 получим

$$
\frac{1}{T} \int_{1}^{T} Q_{\alpha}^{(t)} \widetilde{x} d t \rightarrow \varphi \otimes \lambda(\widetilde{x}) 1_{\mathfrak{A} \otimes \mathfrak{A}} \quad \text { при } \quad T \rightarrow \infty, \quad \widetilde{x} \in \mathfrak{M} .
$$

Пусть $f$ - произвольная функция из $C(Z)$ и $x \in \mathfrak{A}$. Согласно определению к.к.с.п. $Q_{\alpha}^{(t)}$ имеем

$$
\left(Q_{\alpha}^{(t)}(x \otimes f)\right)(\mathbf{x}, \mathbf{y})=\left(P_{\alpha}^{(t)} f\right)(\mathbf{x}, \mathbf{y}) P^{(t)} x, \quad \mathbf{x}, \mathbf{y} \in Z
$$

В силу (2.12) получим

$$
\frac{1}{T} \int_{1}^{T}\left(P_{\alpha}^{(t)} f\right)(\mathbf{x}, \mathbf{y}) P^{(t)} x d t \rightarrow \lambda(f) \varphi(x) 1_{\mathfrak{A}} \quad \text { при } \quad T \rightarrow \infty, \quad x \in \mathfrak{A}
$$

Теорема 2.3. Пусть $P^{(t)}$ - строго перемешивающий к.к.с.п. на $\mathcal{A}$. Тогда для каждых $x \in \mathfrak{A}$ и Ф-функиии Безиковича $b(t)$ средние

$$
\frac{1}{T} \int_{1}^{T} b(t) P^{(t)} x d t
$$

сходятся равномерно в $\mathfrak{A} \otimes \mathfrak{A}$.

ДокаЗАТЕЛьство. Пусть $\varepsilon>0$-произвольное число, $x \in \mathfrak{A}$ и $b(t)-\Phi$-функция Безиковича. Тогда существуют набор $\alpha=\left\{\alpha_{i}\right\}_{i=1}^{s} \in F$, функция $f \in C(Z)$ и $\mathbf{x}, \mathbf{y} \in Z$ такие, что справедливо (2.11). Имеем

$$
\begin{aligned}
& \left\|\frac{1}{T} \int_{1}^{T} b(t) P^{(t)} x d t-\frac{1}{T} \int_{1}^{T}\left(P_{\alpha}^{(t)} f\right)(\mathbf{x}, \mathbf{y}) P^{(t)} x d t\right\| \\
& \quad \leqslant \frac{1}{T} \int_{1}^{T}\left|b(t)-\left(P_{\alpha}^{(t)} f\right)(\mathbf{x}, \mathbf{y})\right|\left\|P^{(t)} x\right\| d t \leqslant\|x\| \varepsilon
\end{aligned}
$$

Из (2.13) следует, что средние (2.14) сходятся равномерно в $\mathfrak{A} \otimes \mathfrak{A}$. Теорема доказана.

ЗАмечАниЕ. Аналогичная теорема была доказана в [14], [15] для положительных отображений алгебр фон Неймана с весом - последовательностью Безиковича в квазиравномерной топологии. 


\section{Список литературы}

1. Бернштейн C.H. Решение одной математической проблемы, связанной с теорией наследственности // Уч. записки НИ кафедр Украины. 1924. №1. С. 83-115.

2. Улам C. Нерешенные математические задачи. М.: Наука, 1964.

3. Kesten $H$. Quadratic transformations: a model for population growth. I, II // Adv. Appl. Prob. 1970. V. 2. P. 1-82; 1970. V. 2. P. 179-228.

4. Любич Ю. И. Основные понятия и теоремы эволюционной генетики свободных популяций // УМН. 1971. Т. 26. № 5. С. 51-116.

5. Сарымсаков Т. А., Ганиходжаев Р. Н. Эргодический принцип для квадратичных стохастических операторов // Изв. АН УзССР. Сер. физ.-матем. наук. 1979. №6. С. 34-38.

6. Ганиходжаев Н. Н., Мухамедов Ф. М. О квантовых квадратичных стохастических процессах // Докл. АН РУз. 1997. № 3. С. 13-16.

7. Ганиходжсев Н. Н., Мухамедов Ф. М. О квантовых квадратичных стохастических процессах и некоторые эргодические теоремы для таких процессов // Узб. матем. журн. 1997. № 3. C. 8-20.

8. Sakai S. $C^{*}$-algebras and $W^{*}$-algebras. Berlin: Springer-Verlag, 1971.

9. Любич Ю.И. Математические структуры в популяционной генетике. Киев: Наукова думка, 1983.

10. Janks R. D. Quadratic differential systems for interactive population models // J. Differential Equations. 1969. V. 5. № 3. P. 497-514.

11. Сарымсаков T. А., Ганиходжсаев H. Н. Аналитические методы в теории квадратичных стохастических операторов // Докл. АН СССР. 1989. Т. 305. № 5. С. 1052-1056.

12. Сарымсаков Т. А., Ганиходжаев Н. Н. Об эргодическом принципе для квадратичных процессов // Докл. АН СССР. 1991. Т. 316. №6. С. 1315-1319.

13. Корнфельд Н.П., Синай Я.Г., Фомин С. В. Эргодическая теория. М.: Наука, 1980.

14. Hensz E. On some ergodic theorems for von Neumann algebras // Probability theory on vector spaces. III. Proc. Conf., Lublin/Pol. 1983. Lecture Notes in Math. V. 1080, 1984. P. 119-123.

15. Litvinov S. On weighted ergodic theorems in von Neumann algebras (to appear).

Институт математики им. В.И. Романовского АН РУЗ, Ташкент
Поступила в редакцию 06.05 .1998 и 25.01 .1999 